\title{
Evolução nutricional de pacientes com transtornos alimentares: experiência de 30 anos de um Hospital Universitário ${ }^{1}$
}

\author{
Nutritional status changes in eating disorder \\ patients: 30 years' experience \\ in a University Hospital
}

Raphaela Fernanda Muniz PALMA²

José Ernesto dos SANTOS 3

Rosane Pilot Pessa RIBEIRO 4

RE S U M O

\section{Objetivo}

Descrever a evolução do estado nutricional e dos parâmetros bioquímicos de pacientes com anorexia nervosa e bulimia nervosa internados para tratamento durante seguimento em serviço especializado.

\section{Métodos}

Foram revisados todos os prontuários dos pacientes em seguimento pelo serviço de 1982 a 2011 , que foram internados para tratamento. Dados sociodemográficos, peso, altura e exames bioquímicos foram coletados no início e final da internação.

\section{Resultados}

No período referido, 83 pacientes com diagnóstico de anorexia ou bulimia nervosa foram internados para tratamento. A maioria era do sexo feminino $(95,2 \%)$ raça branca $(94,0 \%)$, solteira $(76,0 \%)$ e sem filhos $(78,3 \%)$. O diagnóstico predominante foi de anorexia nervosa (85,5\%), sobretudo em seu tipo restritivo (54,2\%). 0 índice de massa corporal médio dos pacientes adultos $(n=41)$ evoluiu de $16,53 \pm 4,9$ para $17,47 \pm 4,3 \mathrm{~kg} / \mathrm{m}^{2}$

1 Artigo elaborado a partir da dissertação de RFM PALMA, intitulada "Hospitalização integral para o tratamento de transtornos alimentares: características e resultados”. Universidade de São Paulo; 2012. Apoio: Fundação de Amparo à Pesquisa do Estado de São Paulo (Processo no 2010/02417-8).

2 Universidade de São Paulo, Escola de Enfermagem de Ribeirão Preto, Programa de Pós-Graduação de Enfermagem em Saúde Pública. Av. Bandeirantes, 3900, Monte Alegre, 14049-900, Ribeirão Preto, SP, Brasil. Correspondência para/Correspondence to: RFM PALMA. E-mail: <rapha_muniz@yahoo.com.nr>.

${ }^{3}$ Universidade de São Paulo, Faculdade de Medicina de Ribeirão Preto, Departamento de Clínica Médica. Ribeirão Preto, SP, Brasil.

${ }^{4}$ Universidade de São Paulo, Escola de Enfermagem de Ribeirão Preto, Departamento de Enfermagem Materno-Infantil e Saúde Pública. Ribeirão Preto, SP, Brasil. 
$(p<0,05)$. Para as crianças e adolescentes $(n=38)$, passou de $13,78 \pm 2,57$ para $15,11 \pm 2,99 \mathrm{~kg} / \mathrm{m}^{2}(p<0,05)$. Os valores médios da maioria dos parâmetros bioquímicos estavam dentro do limite da normalidade e, apenas o betacaroteno, manteve-se em níveis médios acima da normalidade no início e final da internação. Os valores de albumina, hematócrito, lipoproteína de alta densidade e potássio melhoraram ao final da internação $(p<0,05)$.

\section{Conclusão}

A hospitalização integral, apesar das dificuldades e da complexidade inerentes ao tratamento dos transtornos alimentares, considerando-se principalmente a resistência frente à recuperação do peso, pode proporcionar melhoria no estado nutricional e normalização de diversos parâmetros bioquímicos.

Termos de indexação: Anorexia nervosa. Bulimia nervosa. Hospitalização. Transtornos alimentares.

\section{A B S TR A C T}

\section{Objective}

To describe the changes in the nutritional status and biochemical parameters of patients with anorexia nervosa and bulimia nervosa during inpatient treatment at a specialized service.

\section{Methods}

We reviewed the medical records of all patients followed by the service from 1982 to 2011 who had been hospitalized for treatment. Sociodemographic data, weight, height, and biochemical tests were collected at hospital admission and discharge.

\section{Results}

During the period, 83 patients diagnosed with anorexia or bulimia nervosa had been hospitalized for treatment. Most were female (95.2\%), white (94.0\%), single (76.0\%), and childless (78.3\%). The predominant diagnosis was anorexia nervosa (85.5\%), especially the restrictive type (54.2\%). The body mass index of adult patients $(n=41)$ increased from $16.53 \pm 4.9$ to $17.47 \pm 4.3 \mathrm{~kg} / \mathrm{m}^{2}(p<0.05)$, and of children and adolescents $(n=38)$, from $13.78 \pm 2.57$ to $15.11 \pm 2.99 \mathrm{~kg} / \mathrm{m}^{2}(p<0.05)$. The mean values of most biochemical parameters were within the normal limits, and only the mean beta-carotene levels were elevated at admission and discharge. Albumin, hematocrit, high-density lipoprotein, and potassium levels were higher at discharge $(p<0.05)$.

\section{Conclusion}

Despite the difficulties and complexities inherent to the treatment of eating disorders, especially patients' resistance to weight recovery, inpatient treatment can improve nutritional status and normalize several biochemical parameters.

Indexing terms: Anorexia nervosa. Bulimia nervosa. Hospitalization. Eating disorders.

\section{N T R O D U Ç Ã O}

Os Transtornos Alimentares (TA) mais discutidos são a Anorexia Nervosa (AN) e a Bulimia Nervosa (BN), cuja prevalência é variável em estudos epidemiológicos devido à divergência entre instrumentos, metodologia e/ou utilização de critérios diagnósticos mais restritos ou abrangentes. Varia entre 0,3 e 3,7\% na $A N$ e na BN entre 1,0 e 4,2\% ${ }^{1-4}$. Em relação ao sexo ${ }^{5}$, os TA apresentam predominância em mulheres jovens, com relação homem-mulher em média de 1:10 e até de 1:20. No Brasil, não existem estudos epidemiológicos com bases populacionais represen- tativas, utilizando-se instrumentos adequados e validados para se investigar a prevalência de TA.

Os transtornos alimentares influenciam intensamente o estado nutricional e o metabolismo. Suas conseqüências físicas se refletem sobre vários sistemas orgânicos podendo ocorrer alterações endócrinas, hidroeletrolíticas, gastrintestinais, alterações no sistema imunológico com redução dos mecanismos de defesa, diminuição da força muscular e acometimento do músculo cardíaco. As complicações clínicas relacionadas à AN são decorrentes diretamente da desnutrição, enquanto que na BN estão frequentemente relacionadas aos distúrbios hidroeletrolíticos ${ }^{6}$. 
Há evidências de que, com a restauração do peso, muitas das complicações clínicas dos TA podem ser revertidas ${ }^{7-9}$. Sendo assim, a estabilização dos parâmetros clínicos e nutricionais são componentes essenciais para o tratamento dos TA. Dada a complexidade da etiologia dos TA, torna-se imprescindível o olhar atento e multifacetado para o manejo terapêutico da $\mathrm{AN}$ e $\mathrm{BN}$; o tratamento dessas doenças é desafiador e requer a abordagem de uma equipe multidisciplinar bem treinada em serviços especializados.

O nível de cuidado apropriado para um paciente é determinado no momento do diagnóstico inicial e, posteriormente, sempre que uma mudança relevante em sua condição exija uma transição para um nível diferente. As configurações incluem o seguimento ambulatorial, tratamento domiciliar, hospitalização parcial em Hospitais-Dia (HD) e hospitalização integral para pacientes graves que necessitem de tratamento para as complicações agudas da doença.

Inicialmente, o tratamento deve ocorrer em contexto ambulatorial com equipe multidisciplinar que objetivaria, em primeira instância, a normalização do comportamento e padrão alimentar, bem como a recuperação do peso. A hospitalização parcial geralmente ocorre em HD, sendo indicada para pacientes que não necessitam de supervisão e cuidados 24 horas, mas que também não são capazes de apresentar sucesso nos programas ambulatoriais. Nesse nível os pacientes recebem atendimento e supervisão por um período de até 8 horas, com atividades estruturadas incluindo apoio e incentivo durante as refeições e lanches. Já a hospitalização integral é a modalidade terapêutica utilizada pelas equipes quando outras tentativas de tratamento falharam. Ocorre em serviços especializados sob regime de internação em hospitais de nível terciário ou hospitais universitários. As indicações para hospitalização integral de pacientes com TA, em geral, englobam: estado nutricional insatisfatório, descompensação clínica com distúrbios hidroeletrolíticos, bradicardia, hipotensão, comorbidades psiquiátricas ou risco de suicídio iminente, pouca continência familiar e situações de tensão ambiental, abuso de substâncias e exacerbação de sintomas purgativos ${ }^{10,11}$.

São poucos os estudos e dados na literatura internacional e nacional referentes aos resultados do tratamento com hospitalização integral de pacientes portadores de AN ou BN. Além disso, no Brasil existem poucos serviços especializados na assistência aos TA, o que contribui para a escassez de dados sobre o tema.

O objetivo do presente estudo foi descrever a evolução do estado nutricional e parâmetros bioquímicos de pacientes com $\mathrm{AN}$ ou BN internados para tratamento durante seguimento em serviço especializado.

\section{M É T O D O S}

Trata-se de um estudo longitudinal de caráter descritivo e comparativo, com delineamento quantitativo.

No início dos anos 1980, foi criado pela Divisão de Nutrologia, do Departamento de Clínica Médica, da Faculdade de Medicina de Ribeirão Preto, o Grupo de Assistência em Transtornos Alimentares (GRATA), para prestar assistência aos portadores de TA no Hospital das Clínicas da Faculdade de Medicina de Ribeirão Preto, da Universidade de São Paulo (HCFMRP-USP). Ao longo dos anos, à equipe foram agregados vários profissionais e, atualmente, conta-se com uma equipe multidisciplinar composta por médicos (nutrólogo e psiquiatra), psicólogos e nutricionistas, além de estagiários nas áreas de Psicologia e Nutrição' ${ }^{12}$.

Foram incluídos no estudo os pacientes com diagnóstico de $\mathrm{AN}$ ou BN seguidos pelo GRATA, que foram internados entre 1982 e 2011 - ano em que consta a primeira internação realizada pelo grupo e última de acordo com o período de coleta de dados -, independente do sexo e idade, com duração de internação igual ou superior a três dias. Foram excluídos do estudo pacientes com internação inferior a data descrita, pelo fato de não ser possível mensurar possível benefícios 
no estado nutricional, além dos pacientes que apesar do diagnóstico do TA, não receberam atendimento pelo GRATA ou cujos prontuários não foram encontrados nos arquivos médicos.

Por meio do levantamento dos registros de pacientes realizado em sistema informatizado implantado no HCFMRP-USP junto ao Departamento de Seção de Dados Médicos, foram encontrados os registros de 171 pacientes que estiveram internados com diagnóstico de AN ou BN no referido período. A partir da localização e pesquisa em cada um dos 171 prontuários, 88 pacientes foram excluídos do estudo (30 pacientes não eram portadores de TA, 39 pacientes receberam atendimento por outra especialidade médica que não o GRATA, 14 pacientes tiveram tempo de hospitalização inferior a três dias e cinco prontuários não foram encontrados). Sendo assim, a amostra final foi constituída por dados referentes a 83 pacientes portadores de TA que foram atendidos pelo GRATA e que necessitaram de internação para tratamento de $A N$ ou BN.

Os dados foram coletados a partir da revisão dos prontuários dos pacientes, seguindo o instrumento de coleta desenvolvido para a pesquisa. Foram colhidos dados sociodemográficos, peso, altura e resultados de exames bioquímicos (albumina, proteínas totais, ferro, hemoglobina, hematócrito, glicemia, colesterol, triglicérides, frações Low Density Lipoprotein (LDL) e High Density Lipoprotein-cholesterol (HDL-colesterol), sódio, potássio, vitaminas $\mathrm{A}, \mathrm{C}, \mathrm{B}_{12}$, beta-caroteno e ácido fólico) no início e final da internação daqueles internados apenas uma vez. Para aqueles internados mais vezes, os dados foram coletados no início da primeira internação e no final da última internação. Assim, pode-se comparar a evolução da hospitalização em geral, e não de cada interação separadamente. A adequação dos exames bioquímicos seguiu os valores de referência adotados pelo Laboratório de Análises Clínicas do HCFMRP-USP.

A determinação do estado nutricional de pacientes adultos ( $\geq 20$ anos) foi feita mediante classificação do Índice de Massa Corporal (IMC), segundo preconizado pela Organização Mundial da Saúde ${ }^{13}$. Para crianças e adolescentes até 19 anos a classificação seguiu a estratificação segundo percentuais obtidos pela curva de IMC para idade utilizada pelo Sistema de Vigilância Alimentar e Nutricional ${ }^{14}$.

As informações coletadas foram transportadas para um banco de dados com técnica de dupla-digitação e validação no programa Excel, do software Microsoft Office, versão 2007. Os resultados descritivos foram obtidos por meio do cálculo das médias (desvio-padrão), valores mínimos e máximos para as variáveis contínuas, além das frequências para as variáveis categóricas. A normalidade das variáveis contínuas foi avaliada por meio do teste Kolmogorov-Smirov. Foram empregados os testes paramétricos $t$ Student pareado e análise de variância (Anova). Para verificar diferenças estatísticas entre as múltiplas comparações no teste Anova, optou-se pelo método Bonferroni. O teste não-paramétricos Wilcoxon foi utilizado para verificar a evolução da glicemia que não apresentou distribuição normal. O valor de $p<0,05$ foi adotado como significante e todos os cálculos das análises estatísticas foram realizados com auxílio do software Statistical Package for Social Sciences (SPSS) versão 17.0 (SPSS Inc. (hicago, USA).

A pesquisa foi aprovada pelo Comitê de Ética em Pesquisa do HCFMRP-USP (n 976/2010).

\section{RESULTADOS}

No período de abrangência do estudo, o GRATA prestou atendimento ambulatorial para 186 pacientes com $T A$, sendo que 83 deles $(44,6 \%)$ necessitaram de, no mínimo, uma internação durante o tratamento. A média do número de inter-

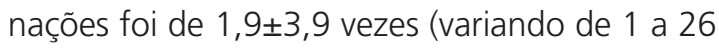
vezes).

Os pacientes em sua maioria eram do sexo feminino ( $n=79 ; 95,2 \%)$ e da raça branca $(n=78$; $94,0 \%)$, com média de idade na época do

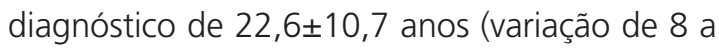
58 anos), sendo $53,0 \%$ adultos ( $n=44$ ) e $47,0 \%$ 
crianças e adolescentes $(n=39)$. A maioria era solteira ( $n=63 ; 76,0 \%)$, sem filhos ( $n=65 ; 78,3 \%$ ) e cerca da metade dos pacientes era estudante cursando o ensino médio ( $n=42 ; 50,6 \%$ ). Observa-se que a maior parte deles reside em cidades do estado de São Paulo ( $n=73 ; 88,0 \%$ ) sendo, aproximadamente um terço, moradores de Ribeirão Preto ( $n=30 ; 36,1 \%)$.

O diagnóstico demonstrou preponderância de AN ( $n=71 ; 85,5 \%)$. Quando observado os seus subtipos, cerca de quase metade $(n=45$; 54,2\%) apresentava Anorexia Nervosa do tipo Restritivo (AN-R) e 31,3\% ( $n=26)$ tinha Anorexia Nervosa do tipo Compulsão Periódica/Purgativo
(AN-CP). Pacientes portadores de BN abarcaram apenas $14,5 \%(n=12)$ dos casos.

Os valores médios do IMC - segundo diagnóstico (Tabela 1) -, revelaram que os pacientes com AN-R mantiveram-se ao término da internação com magreza grau III. Houve transição ao término da internação para os pacientes portadores de $\mathrm{AN}-\mathrm{CP}$, evoluindo de magreza grau III para grau II. Nos subtipos de AN, evidencia-se um aumento nos valores finais de IMC, o que não ocorreu entre os pacientes com BN - apesar de permanecerem, em média, em eutrofia, tanto no início quanto no término da internação. Houve diferença estatística entre os valores médios de

Tabela 1. Índice de massa corporal $\left(\mathrm{kg} / \mathrm{m}^{2}\right)$ dos pacientes hospitalizados no início e final das internações segundo diagnóstico. Ribeirão Preto (SP), 2011.

\begin{tabular}{|c|c|c|c|c|c|c|c|c|c|c|}
\hline \multirow{2}{*}{ Diagnóstico } & \multicolumn{5}{|c|}{ Início* } & \multicolumn{5}{|c|}{ Final $^{* *}$} \\
\hline & $n$ & M & DP & Min & Max & $n$ & M & DP & Min & Max \\
\hline AN-R & 45 & 13,5 & 2,5 & 8,9 & 21,6 & 44 & 14,8 & 2,7 & 09,3 & 22,1 \\
\hline$A N-C P$ & 26 & 15,7 & 3,9 & 9,1 & 21,2 & 25 & 16,9 & 2,9 & 11,9 & 23,3 \\
\hline BN & 12 & 22,0 & 5,4 & 16,1 & 37,3 & 10 & 21,0 & 6,3 & 13,8 & 32,7 \\
\hline
\end{tabular}

Nota: "AN-R < AN-CP e BN $(p<0,05) ;{ }^{* *} A N-R<$ BN e AN-CP < BN (p<0,05); M: Média; DP: Desvio-Padrão; Mín: Mínimo; Máx: Máximo; AN-R: Anorexia Nervosa do tipo Restritivo; AN-CP: Anorexia Nervosa do tipo Compulsão Periódica/Purgativo; BN: Bulimia Nervosa.

Tabela 2. Parâmetros bioquímicos dos pacientes portadores de transtorno alimentar hospitalizados no HCFMRP-USP no início e final das internações. Ribeirão Preto (SP), 2011.

\begin{tabular}{|c|c|c|c|c|c|c|}
\hline \multirow{2}{*}{ Parâmetro bioquímico } & \multirow{2}{*}{$\mathrm{n}$} & \multicolumn{2}{|c|}{ Inicial } & \multicolumn{2}{|c|}{ Final } & \multirow{2}{*}{$p$} \\
\hline & & M & DP & M & DP & \\
\hline Albumina ( $\mathrm{g} / \mathrm{dL}$ ) & 49 & 3,82 & 0,71 & 4,16 & 0,71 & 0,000 \\
\hline Proteínas totais ( $\mathrm{g} / \mathrm{dL}$ ) & 30 & 6,74 & 0,89 & 6,78 & 0,94 & 0,848 \\
\hline Ferro $(\mathrm{mg} / \mathrm{dL})$ & 29 & 75,06 & 33,85 & 86,01 & 33,92 & 0,234 \\
\hline Hemoglobina (g\%) & 42 & 12,40 & 1,61 & 12,22 & 1,49 & 0,474 \\
\hline Hematócrito (\%) & 42 & 34,71 & 2,97 & 36,80 & 4,21 & 0,000 \\
\hline Glicemia (mg/dL) & 45 & 81,85 & 42,44 & 82,75 & 19,29 & 0,340 \\
\hline Colesterol (mg/dL) & 19 & 154,38 & 35,62 & 158,05 & 35,09 & 0,725 \\
\hline Triglicérides (mg/dL) & 19 & 81,26 & 43,88 & 71,68 & 35,96 & 0,413 \\
\hline LDL-colesterol (mg/dL) & 9 & 86,56 & 30,89 & 85,56 & 25,19 & 0,948 \\
\hline HDL-colesterol (mg/dL) & 16 & 45,61 & 9,98 & 51,48 & 12,44 & 0,017 \\
\hline Potássio (mmol/L) & 51 & 3,83 & 0,71 & 4,20 & 0,44 & 0,003 \\
\hline Sódio (mEq/L) & 51 & 139,06 & 4,56 & 140,04 & 3,29 & 0,169 \\
\hline Vitamina A (ug/dL) & 15 & 46,26 & 26,86 & 46,48 & 25,67 & 0,982 \\
\hline Vitamina C (mg/dL) & 16 & 0,34 & 0,21 & 0,38 & 0,15 & 0,548 \\
\hline Vitamina $B_{12}(p g / m L)$ & 7 & 518,29 & 328,52 & 455,14 & 36,66 & 0,642 \\
\hline Beta caroteno (ug/dL) & 15 & 210,21 & 166,60 & 199,04 & 111,70 & 0,702 \\
\hline Ácido fólico (ng/mL) & 8 & 11,26 & 7,02 & 11,23 & 5,27 & 0,990 \\
\hline
\end{tabular}

Nota: M: Média; DP: Desvio-Padrão; LDL: Low Density Lipoprotein (lipoproteína de baixa densidade); HDL: High Density Lipoprotein (lipoproteína de alta densidade); HCFMRP-USP: Hospital das Clínicas da Faculdade de Medicina de Ribeirão Preto da Universidade de São Paulo. 
IMC inicial e entre os diferentes diagnósticos. Já para o IMC final, as diferenças foram observadas entre os pares de grupos AN-R e BN; e também AN-CP e BN $(p<0,05)$.

O IMC médio dos pacientes adultos $(n=41)$ no início da internação foi de $16,53 \pm 4,9 \mathrm{~kg} / \mathrm{m}^{2}$ (mínimo:9,1; máximo $37,3 \mathrm{~kg} / \mathrm{m}^{2}$ ) e ao final

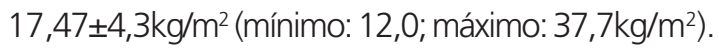
Para as crianças e adolescentes $(n=38)$, o valor médio encontrado para o IMC inicial foi de 13,78kg $/ \mathrm{m}^{2} \pm 2,57 \mathrm{~kg} / \mathrm{m}^{2}$ (mínimo:8,9kg/m²; máximo: $\left.19,4 \mathrm{~kg} / \mathrm{m}^{2}\right)$; ao final da internação a média foi de $15,11 \mathrm{~kg} / \mathrm{m}^{2} \pm 2,99 \mathrm{~kg} / \mathrm{m}^{2}$ (mínimo: 9,3; máximo: $\left.23,3 \mathrm{~kg} / \mathrm{m}^{2}\right)$. Apesar de continuarem abaixo

Tabela 3. Valores de referência de exames bioquímicos adotados pelo HCFMRP-USP. Ribeirão Preto (SP), 2011.

\begin{tabular}{|c|c|}
\hline Parâmetro bioquímico & Valor de referência \\
\hline Albumina & $3,5-5 \mathrm{~g} / \mathrm{dL}$ \\
\hline Proteínas totais & $6,4-8,2 \mathrm{~g} / \mathrm{dL}$ \\
\hline Ferro & $35-150 \mathrm{mg} / \mathrm{dL}$ \\
\hline \multirow[t]{2}{*}{ Hemoglobina } & $13,5-17,5 \mathrm{~g} \%$ \\
\hline & $12-15,5 g \%$ \\
\hline \multirow[t]{2}{*}{ Hematócrito } & $\hat{\delta} 39-45 \%$ \\
\hline & $35-45 \%$ \\
\hline Glicemia & $70-100 \mathrm{mg} / \mathrm{dL}$ \\
\hline Colesterol & $<200 \mathrm{mg} / \mathrm{dL}$ \\
\hline Triglicérides & $<150 \mathrm{mg} / \mathrm{dL}$ \\
\hline LDL-C & $<130 \mathrm{mg} / \mathrm{dL}$ \\
\hline HDL-C & $>40 \mathrm{mg} / \mathrm{dL}$ \\
\hline Sódio & $135-145 \mathrm{mEq} / \mathrm{L}$ \\
\hline Potássio & $3,5-5 \mathrm{mmol} / \mathrm{L}$ \\
\hline \multirow[t]{4}{*}{ Vitamina A } & Deficiente $10 \mathrm{ug} / \mathrm{dL}$ \\
\hline & Baixo 10 - 19ug/dL \\
\hline & Aceitável 20 - 49ug/dL \\
\hline & Alto $>50 u g / d L$ \\
\hline \multirow[t]{3}{*}{ Vitamina C } & Deficiente $<0,2 \mathrm{mg} / \mathrm{dL}$ \\
\hline & Baixo 0,2 - 0,29mg/dL \\
\hline & Aceitável 0,3mg/dL \\
\hline Vitamina $B_{12}$ & $174-878 p g / m L$ \\
\hline Ácido fólico & $3-17 n g / d L$ \\
\hline \multirow[t]{4}{*}{ Betacaroteno } & Deficiente $<20 \mathrm{ug} / \mathrm{dL}$ \\
\hline & Baixo 20 - 30ug/dL \\
\hline & Aceitável 40 - 99ug/dL \\
\hline & Alto $\geq 100 u g / d L$ \\
\hline
\end{tabular}

Nota: HCFMRP-USP: Hospital das Clínicas da Faculdade de Medicina de Ribeirão Preto da Universidade de São Paulo; LDL-c: Low Density Lipoprotein-cholesterol (lipoproteína de baixa densidade-colesterol); HDL-c: High Density Lipoprotein-cholesterol (lipoproteína de alta densidade-colesterol) dos valores referentes à eutrofia, a melhoria observada na evolução do estado nutricional durante a hospitalização de adultos, crianças e adolescentes demonstrou diferença estatística $(p<0,05)$.

Os valores médios da maioria dos parâmetros bioquímicos avaliados (albumina, proteínas totais, ferro, hemoglobina, hematócrito, glicemia, colesterol total, triglicérides, LDL-colesterol, HDH-colesterol, potássio, sódio, vitamina $\mathrm{C}$, vitamina $A$, vitamina $B_{12}$ e ácido fólico) estavam dentro do limite da normalidade (Tabelas 2 e 3). Apenas para o betacaroteno, os níveis médios encontravam-se acima da normalidade no início e final da internação. Destaca-se a melhoria para albumina, hematócrito, HDL-colesterol e potássio ao final da internação $(p<0,05)$.

\section{DIS C USS ÃO}

Estudos têm demonstrado que pacientes diagnosticados com TA, com quadro grave em sua maioria, necessitarão de pelo menos uma internação para tratamento durante o curso da doença na tentativa de reverter ou minimizar as complicações clínicas e comorbidades associadas ${ }^{15}$. Apesar da elevada proporção de internações durante o tratamento, pouco se sabe sobre as características desses pacientes e o tipo de tratamento prestado a eles ${ }^{16}$. Há escassez, sobretudo na literatura nacional, na divulgação dos resultados da hospitalização de pacientes com TA.

As características sociodemográficas dos pacientes hospitalizados pelo GRATA foram semelhantes em relação a outros estudos: predominância de pacientes do sexo feminino e da raça branca, com média de idade na época do diagnóstico próxima aos 21 anos. A maioria era solteira, sem filhos, estudantes cursando o ensino médio e residente da área urbana ${ }^{16-21}$.

A Anorexia nervosa, devido às suas graves consequências nos sistemas orgânicos decorrentes da desnutrição ${ }^{22,23}$, associada à gravidade ainda maior quando diagnosticada em crianças e adolescentes por influenciar diretamente o desenvolvimento e o crescimento ${ }^{24,25}$, torna-se o diagnós- 
tico de TA mais prevalente nas intervenções hospitalares $^{26-28}$. A distribuição dos pacientes internados pelo GRATA de acordo com o diagnóstico, demonstrou preponderância de $A N$, sobretudo em seu subtipo restritivo.

O estado nutricional é um característica clínica imporante a ser considerada nos TA, pois a desnutrição nos pacientes é um achado recorrente na literatura. Chu et al. ${ }^{21}$ divulgaram que a média de IMC dos 62 pacientes na admissão hospitalar pelo ACUTE (Acute Comprehensive Urgent Treatment for Eating Disorders), em Denver nos Estados Unidos, foi de $12,9 \pm 2,0 \mathrm{~kg} / \mathrm{m}^{2} \mathrm{e}$ $13,1 \pm 1,9 \mathrm{~kg} / \mathrm{m}^{2}$ ao final da internação. O estudo randomizado e controlado de Gowers et al. ${ }^{27}$ encontrou em 57 pacientes internados, IMC médio inicial de $15,3 \pm 1,6 \mathrm{~kg} / \mathrm{m}^{2}$. No estudo das coortes de Willer, Thuras e Crow, observou-se, na década de 1970 , média de $13,7 \pm 1,5 \mathrm{~kg} / \mathrm{m}^{2}$ na admissão hos-pitalar e $16,7 \pm 1,4 \mathrm{~kg} / \mathrm{m}^{2}$ ao término; já nos anos $1990,14,6 \pm 1,7 \mathrm{~kg} / \mathrm{m}^{2}$ no início da internação e $17,2 \pm 1,6 \mathrm{~kg} / \mathrm{m}^{2}$ no final. Os resultados dessa investigação demonstram que a evolução da média de IMC de 81 pacientes internados na admissão hospitalar passou de 15,4 $\pm 4,3$ para $16,3 \pm 3,9 \mathrm{~kg} / \mathrm{m}^{2}$ no final das internações. Comparativamente aos estudos acima mencionados, observa-se que os resultados encontrados nessa pesquisa mantém a característica comum de manutenção de desnutrição entre os pacientes ao final das internações, apesar do aumento significativo nos valores médios. Em caráter isolado, as maiores médias de IMC foram encontradas no serviço brasileiro.

Alterações bioquímicas e distúrbios hidroeletrolíticos também são manifestações clínicas bastante comuns nos pacientes com TA, principalmente entre os portadores de $\mathrm{BN}$ ou AN-CP devido aos mecanismos compensatórios para evitar o ganho de peso ${ }^{6}$. No presente estudo, os valores médios inicial e final da maioria dos parâmetros bioquímicos avaliados mantiveram-se dentro do limite da normalidade. Entretanto, alguns deles merecem ser discutidos por manterem estreita relação com características clínicas dos pacientes.
A albumina é uma proteína sérica fundamental para a manutenção da pressão osmótica mantendo a distribuição correta dos líquidos corporais entre o compartimento intra e o extravascular. Em pacientes gravemente desnutridos, como nos casos de AN, suas concentrações podem ser mais baixas, pois o consumo alimentar inadequado associado às baixas reservas proteicas, podem diminuir sua síntese no fígado ${ }^{29}$. Em contrapartida, evidências atuais têm demonstrado que as concentrações séricas de albumina mesmo em pacientes com desnutrição importante, geralmente são normais ${ }^{25,30-32}$. A razão para isto não é muito bem conhecida, mas pesquisadores sugerem que os níveis normais podem refletir a preservação relativa de proteína frente à redução do consumo ou a uma mudança nas concentrações de albumina do meio extravascular para o intravascular ${ }^{29}$. Atualmente, a hipoalbuminemia em pacientes com TA é associada ao mal prognóstico ${ }^{33}$. Os achados do presente estudo corroboram o paradoxo da albumina em concentrações normais nos TA, pois a hipoalbuminemia foi encontrada em apenas 12 pacientes (18,0\%) na admissão hospitalar. O mesmo aconteceu para os resultados sanguíneos de proteínas totais, que também estão associadas ao estado nutricional e reservas corporais. A inadequação desse parâmetro esteve presente em apenas 16 pacientes $(24,2 \%)$.

Nos Transtorno Alimentares, principalmente na $A N$, a hipoglicemia é um achado comum $^{34,35}$, principalmente porque reflete o baixo e irregular consumo de glicose por parte desses pacientes. No entanto, a hipoglicemia grave é um sinal de prognóstico ruim e indicativo de hospitalização imediata ${ }^{34}$. Chu et al. ${ }^{21}$ recentemente revelaram que $44 \%$ dos pacientes hospitalizados no ACUTE, entre 2008 e 2010, para estabilização médica apresentavam, na admissão hospitalar, hipoglicemia. Os achados do presente estudo demonstraram prevalência inferior de hipoglicemia no início da internação (31\%).

A anemia pode estar presente em até $30 \%$ dos casos de $A N$ e BN, sendo geralmente do tipo 
normocítica e normocrômica. Esse quadro pode ser consequência da ingestão insuficiente de ferro, favorecendo o aparecimento da deficiênciado tipo ferropriva. Baixas ingestões de vitamina $\mathrm{B}_{12}$ ou folato também podem contribuir para a instalação de anemia macrocítica nos pacientes ${ }^{36-38}$.

Os exames laboratorias investigados nesse estudo sugerem que o perfil de anemia dos pacientes esteve associado à diminuição dos níveis de hemoglobina, visto que as taxas de inadequação para as concentrações de hemoglobina e hematócrito foram encontradas em 35,5\% e $27,6 \%$ dos pacientes, respectivamente, no início da internação. A anemia ferropriva foi pouco prevalente, pois apenas 2 pacientes estavam com valores de ferro sérico baixo do normal, assim como para os outros tipos de anemia relacionadas às deficiências de vitamina $B_{12}$ e ácido fólico. $A$ inadequação na admissão hospitalar para essas vitaminas ocorreu em apenas um paciente.

A hipercarotenemia é um achado laboratorial bem documentado e frequente na AN, responsável pela coloração alaranjada na pele dos pacientes $^{39}$. Suas concentrações séricas estão aumentadas na AN em relação à população em geral ${ }^{41}$. Estudos têm evidenciado hipercarotenemia em número significativo de indivíduos com TA; sua causa não está muito bem elucidada, mas é frequentemente associada à ingestão excessiva de frutas e vegetais ricas em caroteno, alimentos preferidos para consumo devido ao baixo teor calórico.

Boland et al. ${ }^{40}$ encontraram em amostra de 101 pacientes com AN, prevalência de 62,0\% de hipercarotenemia. Sherman et al. ${ }^{41}$ observaram prevalência menor em sua amostra, apenas 13,0\% dos portadores de TA apresentavam esse quadro, nenhum deles com BN. A prevalência de hipercarotenemia nos pacientes de TA desse estudo foi alta e superior aos estudos acima descritos; $73,2 \%$ no início da internação e $77,3 \%$ ao término da hospitalização.

As pesquisas que adotam como metodologia a coleta de dados por meio de revisão de prontuários apresentam, inevitavelmente, viés importante relacionado à perda ou omissão de informações registradas pelos profissionais de saúde. Essa foi a principal dificuldade encontrada no desenvolvimento deste estudo, cuja perda amostral variou amplamente, de 1,2 a 95,5\%, somado ao fato de que muitos pacientes não tinham os exames iniciais e finais para todos os parâmetros bioquímicos investigados. Apesar das limitações do estudo, os resultados e as informações divulgadas poderão contribuir para a literatura científica ao divulgar os resultados da experiência de um serviço especializado ao longo de seus 30 anos de existência.

\section{O N C L U S Ã O}

O presente estudo demonstrou que apesar das dificuldades encontradas no tratamento, considerando-se principalmente a resistência frente à recuperação clínica de um modo geral e, especialmente, em relação ao peso, a hospitalização integral quando indicada adequadamente pode proporcionar melhora no estado nutricional, apesar de ainda ser insuficiente para evolução dos pacientes à eutrofia. Além disso, constatou-se que ela contribui para a melhoria dos resultados na maioria dos exames bioquímicos ao término da hospitalização, o que, observou-se, eleva significativamente os níveis de albumina, hematócrito, lipoproteína de alta densidade e potássio.

\section{A GRADECIMENTOS}

À Profa. Dra. Cláudia Benedita dos Santos pela assessoria nas análises estatísticas e à Fundação de Amparo à Pesquisa do Estado de São Paulo (FAPESP), pelo auxílio financeiro para execução do projeto (Processo $n^{\circ}$ 2010/02417-8).

\section{REFERÊ NCIAS}

1. Aalto-Setälä T, Marttunen M, Tuulio-Henriksson A, Poikolainen K, Lönnqvist J. One-month prevalence of depression and other DSM-IV disorders among young adults. Psychol Med. 2001; 31(5):791-801. 
2. Hoek HW, van Hoeken D. Review of the prevalence and incidence of eating disorders. Int J Eat Disord. 2003; 34(4):383-96.

3. Hoek HW. Incidence, prevalence and mortality of anorexia nervosa and other eating disorders. Curr Opin Psychiatry. 2006; 19(4):389-94.

4. Hudson J, Hiripi E, Harrison G. Pope HG, Kessler RC. The prevalence and correlates of eating disorders in the National Comorbidity Survey Replication. Biol Psychiatry. 2007; 61(3):348-58.

5. Klein DA, Walsh BT. Eating disorders: Clinical features and pathophysiology. Physiol Behav. 2004; 81(2):359-74.

6. Borges NJBG, Sicchieri JMF, Ribeiro RPP, Marchini JS, Dos Santos JE. Transtornos alimentares: quadro clínico. Medicina. 2006; 39(3):340-8.

7. Hadley SJ, Walsh BT. Gastrointestinal disturbances in anorexia nervosa and bulimia nervosa. Curr Drug Targets CNS Neurol Disord. 2003; 2(1):1-9.

8. Mont L, Castro J, Herreros B, Paré C, Azqueta M, Magriña J, et al. Reversibility of cardiac abnormalities in adolescents with anorexia nervosa after weight recovery. J Am Acad Child Adolesc Psychiatry. 2003; 42(7):808-13.

9. Shamim T, Golden NH, Arden M, Filiberto L, Shenker IR. Resolution of vital sign instability: An objective measure of medical stability in anorexia nervosa. J Adolesc Health. 2003; 32(1):73-7.

10. Anzai N, Lindsey-Dudley K, Bidwell RJ. Inpatient and partial hospital treatment for adolescent eating disorders. Child Adolesc Psychiatr Clin N Am. 2002; 11(2):279-309.

11. Woodside DB. Inpatient treatment and medical management of anorexia nervosa and bulimia nervosa. In: Fairburn CG, Brownell KD, editors. Eating disorders and obesity: A comprehensive handbook. $2^{\text {nd }}$ ed. New York: The Guildford Press; 2002.

12. Dos Santos JE. GRATA: nossa história, trabalho e desafios. Medicina. 2006; 39(3):323-6.

13. Organizacíon Mundial de la Salud. Problemas de la salud de la adolescencia: informe de un comité de expertos de la OMS. Series de informes tecnicos $n^{\circ}$ 308. Genebra: Organizacíon Mundial de la Salud; 1965.

14. Brasil. Ministério da Saúde. Sistema de Vigilância Alimentar e Nutricional (SISVAN). Brasília: MS; 2009 [acesso 2009 set 17]. Disponível em: <http:// nutricao.saude.gov.br/documentos/sisvan_norma_ tecnica_criancas.pdf>.

15. Milos G, Spindler A, Hepp U, Schnyder U. Suicide attempts and suicidal ideation: links with psychiatric comorbidity in eating disorder subjects. Gen Hosp Psychiatry. 2004; 26(2):129-35.
16. Calderon R, Vander Stoep A, Collett B, Garrison MM, Toth K. Inpatients with eating disorders: Demographic, diagnostic, and service characteristics from a nationwide pediatric sample. Int J Eat Disord. 2007; 40(7):622-8.

17. Wiseman CV, Sunday SR, Klapper F, Harris WA, Halmi KA. Changing patterns of hospitalization in eating disorder patients. Int J Eat Disord. 2001; 30(1):69-74.

18. Guarda AS, Pinto AM, Coughlin JW, Hussain S, Haug NA, Heinberg LJ. Perceived coercion and change in perceived need for admission in patients hospitalized for eating disorders. Am J Psychiatry. 2007; 164(1):108-14.

19. Gajewska M, Goryñski P, Boguszewska L, Sowiñska M. Hospitalization of people with eating disorders in Polish general and psychiatric hospitals in years 2003-2007. Przegl Epidemiol. 2009; 63(4):571-7.

20. Lopez-de-Andres A, Carrasco-Garrido P, Hernán dez-Barrera V, Gil-de-Miguel A, Jiménez-Trujillo I, Jiménez-García R. Hospitalization trends in Spanish children and adolescents with eating disorders (1998-2007). Appetite. 2010; 55(1):147-51.

21. Chu ES, Gaudiani JL, Mascolo M, Statland B, Sabel A, Carroll K, et al. ACUTE center for eating disorders. J Hosp Medicine. 2012; 7(4):340-4.

22. Mehler PS, Krantz M. Anorexia nervosa medical issues. J Womens Health (Larchmt). 2003; 12(4): 331-40.

23. Sylvester CJ, Forman SF. Clinical practice guidelines for treating restrictive eating disorder patients during medical hospitalization. Curr Opin Pediatr. 2008; 20(4):390-7.

24. Golden $\mathrm{MH}$. The development of concepts of malnutrition. J Nutr. 2002; 132(7):2117S-22S.

25. Katzman DK. Medical complications in adolescents with anorexia nervosa: A review of the literature. Int J Eat Disord. 2005; 37(Suppl):S52-9.

26. Willer MG, Thuras P, Crow SJ. Implications of the changing use of hospitalization to treat anorexia nervosa. Am J Psychiatry. 2005; 162(12):2374-6.

27. Gowers SG, Clark A, Roberts C, Griffiths A, Edwards $V$, Bryan C, et al. Clinical effectiveness of treatments for anorexia nervosa in adolescents: Randomised controlled trial. Br J Psychiatry. 2007; 191:427-35.

28. Long CG, Fitzgerald KA, Hollin CR. Treatment of chronic anorexia nervosa: A 4-year follow-up of adult patients treated in an acute inpatient setting. Clin Psychol Psychother. 2012; 19(1):1-13.

29. Waterlow JC. On serum albumin in anorexia nervosa. Nutrition. 1996; 12(10):720-1.

30. Smith G, Robinson PH, Fleck A. Serum albumin distribution in early treated anorexia nervosa. Nutrition. 1996; 12(10):677-84. 
31. Haluzík M, Papezová M, Nedvídková J, Kábrt J. Serum leptin levels in patients with anorexia nervosa before and after partial refeeding, relationships to serum lipids and biochemical nutritional parameters. Physiol Res. 1999; 48(3):197-202.

32. Caregaro L, Favaro A, Santonastaso P, Alberino F, Di Pascoli L, Nardi M, et al. Insulin-like growth factor 1 (IGF-1): A nutritional marker in patients with eating disorders. Clin Nutr. 2001; 20(3):251-7.

33. Nova E, Lopez-Vidriero I, Varela P, Toro O, Casas JJ, Marcos AA. Indicators of nutritional status in restricting-type anorexia nervosa patients: A 1-year follow-up study. Clin Nutr. 2004; 23(6):1353-9.

34. Connan F, Lightman S, Treasure J. Biochemical and endocrine complications. Eur Eat Disorders Rev. 2000; 8(2):144-157.

35. Guimarães DBS, Salzano FT, Abreu CN. Indicações para internação hospitalar completa ou parcial. Rev Bras Psiquiatr. 2002; 24(3):60-2.

36. Devuyst O, Lambert M, Rodhain J, Lefebvre C, Coche E. Haematological changes and infectious complications in anorexia nervosa: A case-control study. Q J Med. 1993; 86(12):791-9.
37. Saito MI, Silva LEV. Adolescência: prevenção e risco. In: Cereser MG, Cordás TA, editores. Transtornos alimentares: anorexia nervosa e bulimia. São Paulo: Atheneu; 2001.

38. Assumpção CL, Cabral MD. Complicações clínicas da anorexia nervosa e bulimia nervosa. Rev. Bras de Psiquiatr. 2002; 24(3):29-33.

39. Birmingham CL. Hypercarotenemia. N Engl J Med. 2002; 347(3):222-3.

40. Boland B, Beguin C, Zech F, Desager JP, Lambert M. Serum beta-carotene in anorexia nervosa patients: A case-control study. Int J Eat Disord. 2001; 30(3):299-305.

41. Sherman P, Leslie K, Goldberg E, Rybczynski J, St Louis P. Hypercarotenemia and transaminitis in female adolescents with eating disorders: A prospective, controlled study. J Adolesc Health. 1994; 15(3):205-9.

Recebido em: 4/1/2013 Versão final em: 2/9/2013 Aprovado em: 7/10/2013 